\title{
A Moving Source Localization Method for Distributed Passive Sensor Using TDOA and FDOA Measurements
}

\author{
Zhixin Liu, Yongjun Zhao, Dexiu Hu, and Chengcheng Liu \\ Zhengzhou Institute of Information Science and Technology, Zhengzhou, Henan 86-450002, China \\ Correspondence should be addressed to Dexiu Hu; paper_hdx@126.com
}

Received 30 December 2015; Revised 7 March 2016; Accepted 27 March 2016

Academic Editor: Ding-Bing Lin

Copyright ( 2016 Zhixin Liu et al. This is an open access article distributed under the Creative Commons Attribution License, which permits unrestricted use, distribution, and reproduction in any medium, provided the original work is properly cited.

\begin{abstract}
The conventional moving source localization methods are based on centralized sensors. This paper presents a moving source localization method for distributed passive sensors using TDOA and FDOA measurements. The novel method firstly uses the steepest descent algorithm to obtain a proper initial value of source position and velocity. Then, the coarse location estimation is obtained by maximum likelihood estimation (MLE). Finally, more accurate location estimation is achieved by subtracting theoretical bias, which is approximated by the actual bias using the estimated source location and noisy data measurement. Both theoretical analysis and simulations show that the theoretical bias always meets the actual bias when the noise level is small, and the proposed method can reduce the bias effectively while keeping the same root mean square error (RMSE) with the original MLE and Taylor-series method. Meanwhile, it is less sensitive to the initial guess and attains the CRLB under Gaussian TDOA and FDOA noise at a moderate noise level before the thresholding effect occurs.
\end{abstract}

\section{Introduction}

Passive source location has been the focus of considerable research efforts for many years. It is widely used in many areas including radar, sonar $[1,2]$, microphone arrays $[3,4]$, sensor network [5], and wireless communication [6]. For a stationary emitter, the time-difference-of-arrival (TDOA) of a received signal [7] at a number of separated receivers can be used to obtain the source location estimate. Each TDOA defines a hyperbola in which the emitter must lie. The intersection of the hyperbolae gives the source location estimate $[8,9]$. If there is a relative motion between the emitter and the receivers, frequency-difference-of-arrival (FDOA) measurements should be combined with TDOAs to estimate the source position and velocity accurately. In this paper, we enforce locating the moving source using TDOA and FDOA measurements.

Localization of a moving source using TDOA and FDOA is not a trivial task due to the nonlinear nature of the estimation problem [9-12]. Foy put forward a Taylor-series linearization method which requires a proper initial guess close to the true solution in 1976 [10]. However, a good initial guess is not easy to obtain in practice. In order to avoid it,
Ho et al. proposed a novel two-step weighted least-squares (TSWLS) $[9,12]$ approach to obtain the source position and velocity. Sun et al. applied the total least-squares (TLS) technique [13] to solve this problem.

Considering that the noise components of coefficients are linearly dependent, Yu et al. used the constrained total leastsquares (CTLS) [14, 15] in moving source localization.

Up to now, most passive localization methods using TDOA and FDOA are based on centralized structure, which is shown in Figure 1(a). For the centralized structure, one sensor is chosen as the reference sensor and the others can transmit their original signals to the reference sensor [16]. On the one hand, in Electronic Warfare (EW), the receivers, which intercept signals from the emitter and measure their physical parameters, play a central role in the passive location system [17]. Because the emitter is noncooperating, we can only obtain the TDOA and FDOA. For TDOA-based method, common practice requires precise time-synchronization between sensors. Likewise, for FDOA-based method, precise frequency-locking is required. Obviously, for joint TDOAand FDOA-based methods, both types of synchronization are typically required [18]. If a TDOA- and FDOA-based system 


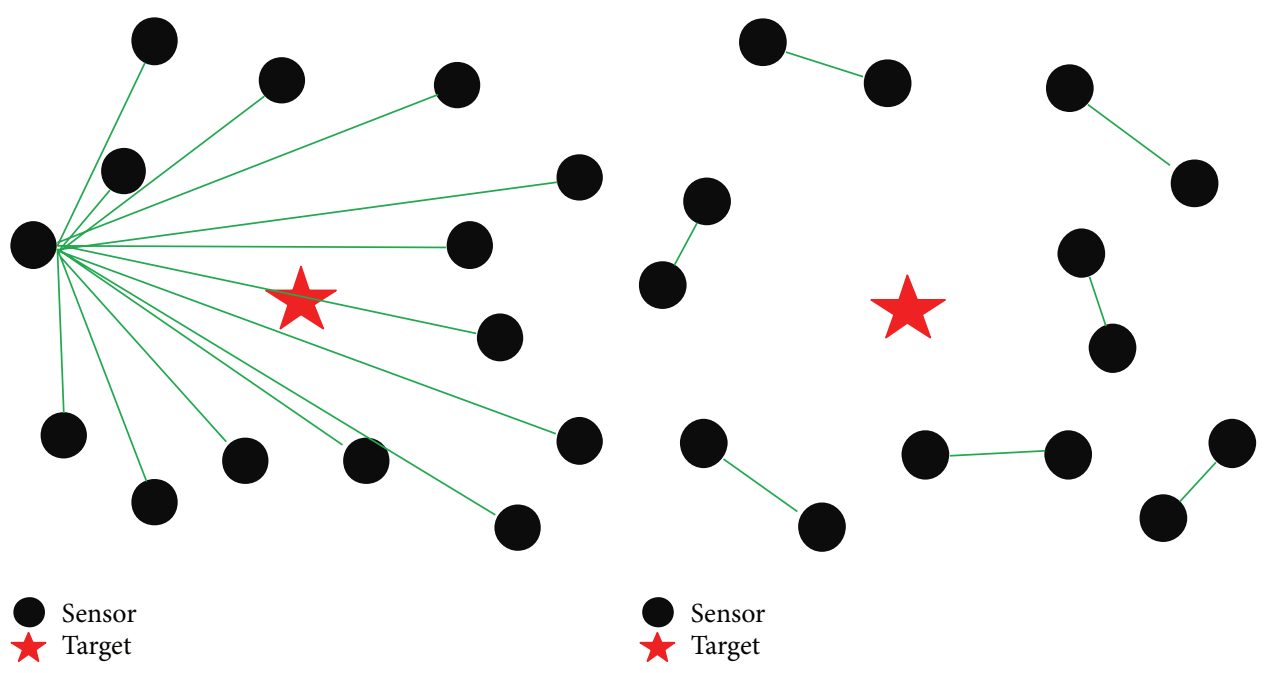

(a) Centralized localization

(b) Distributed localization

FIGURE 1: Sensor pairing.

[18] fails to achieve precise synchronization between devices for separation distance operation, it is impossible to obtain correct measurements from signals sent by receivers, and such a failure to obtain the accurate values directly affects location estimation error [19]. For example, in order to get more accuracy of source position and velocity, the TSWLS, TLS, and CTLS also require precise time-synchronization and frequency-locking among all receivers. The price to pay is accuracy required for TDOA and FDOA measurements, strongly depending on synchronization [17]. Thus, all the receivers would be precisely synchronized in time which is difficult to realize and increase the system complexity.

On the other hand, it is well known that the centralized method is power consuming since raw measurement data is involved in the transmissions and there is high computational cost at a single sensor for centralized localization [20]. In addition, this kind of method requires a high bandwidth which is generally limited in wireless sensor networks (WSNs) and also may cause a large processing delay [21]. This would have significant impacts on the size, weight, and power (SWaP) requirement of that sensor [22].

Therefore, in practice, to reduce the requirements for network bandwidth, synchronization, and power consumption [23], the distributed localization is highly desirable. As seen in Figure 1(b), several sensor pairs are formed without a common reference sensor. The whole sensors do not need to transmit their original signals to the single reference sensor but only to their reference sensor of each group. Furthermore, as the speed of the target is far less than the signal propagation speed and the change of target position in synchronization error is slightly small that we can ignore it, we only need to achieve precise synchronization between two sensors in a group rather than all sensors and have rough synchronization between each group. Therefore, the distributed localization not only reduces the difficulty of the synchronization between all sensors but also decreases the load of data computation and transmission. So, it is necessary to do some deep research into distributed localization problem and improve the estimation accuracy. Nowadays, the distributed structure is a novel problem in passive localization, and there are still some problems for distributed passive localization, such as the optimal sensor pairing strategy, localization algorithm, and optimal baseline between sensors. In this paper, we aim at the location algorithm problem. For conventional centralized structure source location methods, thanks to the only reference sensor in the conventional centralized structure source location methods, they can be transformed from a nonlinear problem to a pseudolinear problem [24]. For instance, $\mathrm{Ho}$ and $\mathrm{Xu}$ used the position and velocity of reference sensor to transform the TDOA and FDOA equation to a set of linear equations and then applied linear weighted least-squares (LS) to obtain the source position [9]. As there are many different reference sensors in distributed structure, it is difficult to use the conventional centralized structure source location methods for estimation. Therefore, it is essential to put forward a distributed localization algorithm.

Meyer et al. put forward the distributed sensor selflocalization and target tracking method in 2012 [25]. Distributed source localization is also a nontrivial problem because the TDOA and FDOA measurements are nonlinearly related to the source location parameters. Due to this problem, the mean square error (MSE) is composed of two parts: the variance and the bias square. When the noise level is low and the observation period is short, the bias is not significant compared with the variance of the target position estimation [26, 27]. Thus, the Cramér-Rao lower bound (CRLB), which is developed for an unbiased estimator, is often used as a reference for evaluating the performance of a location estimator. However, with the increase of observation period, the location variance will decrease and the bias cannot be ignored. It had a serious influence on estimation performance. In particular, with the development of ultrawideband (UWB) technology, the measurements of the target can be measured repeatedly in a short time, and then the precision 
of the target location can be improved by using the method of data fusion [28], but this method can only effectively reduce the variance of the estimation of the target, and the deviation does not decrease as the number of measurements increases $[26,29]$. In application of target tracking, the location deviation has a great influence on performance of target tracking [27]. Therefore, how to remove the deviation from the estimation of target position and velocity is a focus of research. Rui and Ho proposed a bias compensation algorithm [26] based on time of arrival (TOA), TDOA, or angle of arrival (AOA) and verified that the position bias has great influence on location accuracy. In order to reduce this influence, Hao et al. put forward a bias reduction method for passive source localization using TDOA and gain ratios of arrival (GROA) [30]. However, the methods proposed by the above research can only be applied to the stationary target. For moving emitters, a new bias reduction algorithm using both TDOA and FDOA is proposed by Ho [29]. It can reduce the estimation error by adding new constraints to the original position equation. The performance of estimation is the same as maximum likelihood estimation (MLE). However, these methods still have deviation and all need a proper initial guess.

Motivated by the above shortcomings of algorithms based on distributed structure, a new bias compensation method based on MLE for distributed source localization using TDOA and FDOA is proposed in this paper. We study the bias of the MLE [31] for source localization, because the MLE is asymptotically efficient and often serves as a benchmark for performance evaluation. The bias of the MLE of a general estimation problem has been investigated in the mathematical and statistical literature [32-34]. The proposed method firstly uses the classic steepest descent (SD) method $[35,36]$ which has the features of being more rapid to convergence and less sensitive to the initial values to get an appropriate initial guess and coarse location estimation is obtained by MLE $[26,27]$. Then, more accurate location estimation is achieved by subtracting theoretical bias, which is approximated by the actual bias using the estimated source location and noisy data measurement. The proposed method attains the CRLB at a moderate noise level when the TDOA and FDOA noise are Gaussian.

The remainder of the paper is organized as follows. The novel method is introduced in Section 2. Section 3 analyses the CRLB of distributed passive sensors localization under Gaussian measurement noise. Section 4 presents simulation to support the theoretical development of the proposed method. Finally, a brief conclusion is given in Section 5.

\section{The Proposed Algorithm}

2.1. Distributed Localization Model. The model of distributed localization is different from centralized model. We consider a three-dimensional (3D) scenario where an array of $M$ moving sensors is used to determine the position $\mathbf{u}=$ $[x, y, z]^{T}$ and velocity $\dot{\mathbf{u}}=[\dot{x}, \dot{y}, \dot{z}]^{T}$ of a moving source using TDOAs and FDOAs. The sensor position $\mathbf{s}_{i}=\left[x_{i}, y_{i}, z_{i}\right]^{T}$ and velocity $\dot{\mathbf{s}}_{i}=\left[\dot{x}_{i}, \dot{y}_{i}, \dot{z}_{i}\right]^{T}, i=1,2, \ldots, M$, are assumed to be known and $M$ must be even. The location problem requires at least three pairs of receivers (i.e., $M=6$ ) to produce three TDOAs and three FDOAs. This paper focuses on the overdetermined scenario where the number of receivers is larger than 6 . We will use the notation $(*)^{o}$ to denote the true value of the noisy quantity $(*)$.

The Euclidean norm between the source and receiver $i$ is

$$
r_{i}^{o}=\left\|\mathbf{u}^{o}-\mathbf{s}_{i}^{o}\right\|=\sqrt{\left(\mathbf{u}^{o}-\mathbf{s}_{i}^{o}\right)^{T}\left(\mathbf{u}^{o}-\mathbf{s}_{i}^{o}\right)} .
$$

For $M$ sensors, there are a total number of $M / 2$ sensor pairs and TDOA/FDOA measurements. Let

$$
\Sigma=\left\{\{2 i, 2 i-1\} \mid 1 \leq i \leq \frac{M}{2}\right\}
$$

which denotes the set of all sensor pairs. For simplicity and also without loss of generality, the first sensor of each group is chosen as the reference sensor. If the true TDOA of a signal received by the receiver pair $(2 i, 2 i-1)$ is $\tau_{2 i, 2 i-1}^{o}$ and the signal propagation speed is $c$, the set of equations that relates the TDOAs and the source position is

$$
r_{2 i, 2 i-1}^{o}=c \tau_{2 i, 2 i-1}^{o}=r_{2 i}^{o}-r_{2 i-1}^{o},
$$

where $r_{2 i, 2 i-1}^{o}$ is range difference and $i=1,2, \ldots, M / 2$. Note that (3) is nonlinear with respect to $\mathbf{u}$ and the $M / 2$ curves in (3) give the source position estimate. The TDOA equations only allow the estimation of the source position but not velocity. In addition, the TDOA equations alone may not be sufficient to provide enough accuracy to the position estimate.

Due to the moving source, the FDOA measurements can be used to improve the accuracy of position estimate and, at the same time, identify the source velocity. The FDOAs have been converted to the range rate difference through multiplying by signal propagation speed and dividing by the center frequency. Let $\dot{r}_{i}^{o}$ be the true range rate between the source and receiver $i$. By taking the time derivative of (1), it is equal to

$$
\dot{r}_{i}^{o}=\frac{\left(\mathbf{u}^{o}-\mathbf{s}_{i}^{o}\right)^{T}\left(\dot{\mathbf{u}}^{o}-\dot{\mathbf{s}}_{i}^{o}\right)}{r_{i}^{o}} .
$$

The FDOA between receiver pair $2 i$ and $2 i-1$ is the time derivative of (3):

$$
\dot{r}_{2 i, 2 i-1}^{o}=\dot{r}_{2 i}^{o}-\dot{r}_{2 i-1}^{o}
$$

for $i=1,2, \ldots, M / 2$, where $\dot{r}_{2 i, 2 i-1}^{o}$ is range rate difference derived from the FDOAs [9]. Equations (3) and (5) are a set of nonlinear equations with the source position $\mathbf{u}$ and velocity $\dot{\mathbf{u}}$, and solving them from TDOA and FDOA is not an easy task.

In practice, we cannot obtain the true values of TDOA and FDOA. So, we let $\mathbf{r}=\left[r_{2,1}, r_{4,3}, \ldots, r_{M, M-1}\right]^{T}$ and $\dot{\mathbf{r}}=$ $\left[\dot{r}_{2,1}, \dot{r}_{4,3}, \ldots, \dot{r}_{M, M-1}\right]^{T}$ represent the noisy range differences and range rate difference. We will assume that the TDOA and FDOA measurements can be described by the additive noise model as

$$
\begin{aligned}
& \mathbf{r}=\mathbf{r}^{o}+\Delta \mathbf{r}, \\
& \dot{\mathbf{r}}=\dot{\mathbf{r}}^{o}+\Delta \dot{\mathbf{r}},
\end{aligned}
$$


where

$$
\begin{aligned}
& \Delta \mathbf{r}=\left[\Delta r_{2,1}, \Delta r_{4,3}, \ldots, \Delta r_{M, M-1}\right]^{T}, \\
& \Delta \dot{\mathbf{r}}=\left[\Delta \dot{r}_{2,1}, \Delta \dot{r}_{4,3}, \ldots, \Delta \dot{r}_{M, M-1}\right]^{T} .
\end{aligned}
$$

Equation (6) can be written as the function

$$
\mathbf{m}=\mathbf{F}\left(\boldsymbol{\theta}^{o}\right)+\mathbf{n},
$$

where $\mathbf{m}=\left[\mathbf{r}^{T}, \mathbf{r}^{T}\right]^{T}$ is the $M \times 1$ measurement vector with $M / 2$ for range measurement and $M / 2$ for range difference measurement, $\mathbf{F}\left(\boldsymbol{\theta}^{o}\right)=\left[\mathbf{r}^{o T}, \dot{\mathbf{r}}^{o T}\right]^{T}$ represents the function relationship of the noiseless measurement vector in terms of the unknown position $\mathbf{u}^{o}$ and velocity $\dot{\mathbf{u}}^{o}, \boldsymbol{\theta}^{o}=\left[\mathbf{u}^{o T}, \dot{\mathbf{u}}^{o T}\right]^{T}$ is the location and velocity of the source, and $\mathbf{n}=\left[\Delta \mathbf{r}^{T}, \Delta \dot{\mathbf{r}}^{T}\right]^{T}$ is the vectors of TDOA and FDOA noise. They are zero mean Gaussian noise and have covariance matrix $\mathbf{Q}$. We assume the observation interval is long enough so that the measurement noise vectors at different time instants are uncorrelated.

2.2. Initial Solution Using the Steepest Descent Method. Estimating the position and the velocity of a moving emitter using the TDOA/FDOA measurements has been a challenging task because of the high nonlinearity in the TDOA/FDOA signal models. Under some circumstances where the source and receiver geometry is not good, some localization algorithms $[10,26]$ may fail to attain a more accurate estimation without a proper initial guess. In this paper, the appropriate initial value is obtained by SD method $[35,36]$, which features fast convergence speed, low computational complexity, and low requirement of initial value. Although the SD method also requires initial guess, the convergence rate increases as the difference between initial value and true value grows. It is efficient to fill the gap of not having prior knowledge of the target. Therefore, the SD method is chosen as the preferable alternative executing refinement due to its simplicity and validity.

Equation (6) can be expressed as

$$
\begin{aligned}
& \varphi=\mathbf{r}^{o}-\mathbf{r}+\Delta \mathbf{r}, \\
& \boldsymbol{\beta}=\dot{\mathbf{r}}^{o}-\dot{\mathbf{r}}+\Delta \dot{\mathbf{r}},
\end{aligned}
$$

where $\boldsymbol{\varphi}=\left[\varphi_{1}, \varphi_{2}, \ldots, \varphi_{M / 2}\right]^{T}$ and $\boldsymbol{\beta}=\left[\beta_{1}, \beta_{2}, \ldots, \beta_{M / 2}\right]^{T}$. Equation (9) can be written as the function

$$
\Phi=\mathbf{F}\left(\boldsymbol{\theta}^{o}\right)-\mathbf{m}+\mathbf{n},
$$

where $\boldsymbol{\Phi}=\left[\boldsymbol{\varphi}^{T}, \boldsymbol{\beta}^{T}\right]^{T}$. From (10), an objective function is defined as

$$
\rho(\boldsymbol{\theta})=\|\boldsymbol{\Phi}\|^{2}=\sum_{i=1}^{M / 2}\left(\varphi_{i}(\boldsymbol{\theta})\right)^{2}+\sum_{i=1}^{M / 2}\left(\beta_{i}(\boldsymbol{\theta})\right)^{2} .
$$

$\|\cdot\|$ represents the Euclidean norm, and the solution of (6) is a set of unknown variables which is formulated as

$$
\boldsymbol{\theta}=\underset{\boldsymbol{\theta}}{\arg \min } \rho(\boldsymbol{\theta}) .
$$

In general, the gradient direction $\mathbf{G}$ at a certain point is the fastest way to increase the function $\rho(\boldsymbol{\theta})$; on the contrary, the direction of negative gradient $-\mathbf{G}$ is the fastest way to reduce the function value. So, we can use this principle to find $\boldsymbol{\theta}$ that makes (11) minimal. Then, we set an arbitrary initial value denoted by $\boldsymbol{\theta}_{0}=\left[\mathbf{u}_{0}^{T}, \dot{\mathbf{u}}_{0}^{T}\right]^{T}$ and the gradient direction of $\rho(\boldsymbol{\theta})$ at the initial values is defined as

$$
\mathbf{G}_{0}=\left[\begin{array}{ll}
\mathbf{G}_{01}^{T} & \mathbf{G}_{02}^{T}
\end{array}\right]^{T}=\left.\frac{\partial \rho}{\partial \boldsymbol{\theta}}\right|_{\boldsymbol{\theta}_{0}}=\left[\frac{\partial \rho}{\partial \mathbf{u}_{0}^{T}} \frac{\partial \rho}{\partial \dot{\mathbf{u}}_{0}^{T}}\right]^{T} .
$$

We choose the appropriate step size $\lambda, \mu$ to find the new estimation that makes (11) minimal along the direction of the negative gradient $-\mathbf{G}$. So, the new estimation is obtained by

$$
\boldsymbol{\theta}_{1}=\left[\mathbf{u}_{1}^{T}, \dot{\mathbf{u}}_{1}^{T}\right]^{T}=\left[\mathbf{u}_{0}^{T}-\lambda \mathbf{G}_{01}^{T}, \dot{\mathbf{u}}_{0}^{T}-\mu \mathbf{G}_{02}^{T}\right]^{T},
$$

where

$$
\begin{aligned}
& \mathbf{G}_{01}=2 \cdot \sum_{i=1}^{M / 2} \varphi_{i} \cdot \frac{\partial \varphi_{i}}{\partial \mathbf{u}}+2 \cdot \sum_{i=1}^{M / 2} \beta_{i} \cdot \frac{\partial \beta_{i}}{\partial \mathbf{u}}, \\
& \mathbf{G}_{02}=2 \cdot \sum_{i=1}^{M / 2} \beta_{i} \cdot \frac{\partial \beta_{i}}{\partial \dot{\mathbf{u}}}, \\
& \frac{\partial \varphi_{i}}{\partial \mathbf{u}}=\frac{\partial r_{2 i, 2 i-1}}{\partial \mathbf{u}}=\mathbf{x}_{2 i}^{T}-\mathbf{x}_{2 i-1}^{T}, \\
& \frac{\partial \beta_{i}}{\partial \mathbf{u}}=\frac{\partial \dot{r}_{2 i, 2 i-1}}{\partial \mathbf{u}}=\mathbf{v}_{2 i}^{T}-\mathbf{v}_{2 i-1}^{T}, \\
& \frac{\partial \beta_{i}}{\partial \dot{\mathbf{u}}}=\frac{\partial \varphi_{i}}{\partial \mathbf{u}}
\end{aligned}
$$

Appendix A gives details about the evaluation of the derivatives for $\mathbf{x}_{i}$ and $\mathbf{v}_{i}(i=1,2, \ldots, M)$.

In order to make (11) minimal within the shortest steps in new estimation $\boldsymbol{\theta}_{1}$ which is defined as

$$
\begin{aligned}
\rho\left(\boldsymbol{\theta}_{1}\right) & =\rho\left(\mathbf{u}_{1}^{T}, \dot{\mathbf{u}}_{1}^{T}\right) \\
& \approx \min \left\{\rho\left(\mathbf{u}_{0}^{T}-\lambda \mathbf{G}_{01}^{T}, \dot{\mathbf{u}}_{0}^{T}-\mu \mathbf{G}_{02}^{T}\right)\right\},
\end{aligned}
$$


we should find an optimal step size $\lambda$ and $\mu$. Therefore, the Taylor-series expansion of $\rho\left(\boldsymbol{\theta}_{1}\right)$ at $\boldsymbol{\theta}_{0}$ up to second order is

$$
\begin{aligned}
& \rho\left(\boldsymbol{\theta}_{1}\right)=\sum_{i=1}^{M / 2}\left(\varphi_{i}\left(\boldsymbol{\theta}_{1}\right)\right)^{2}+\sum_{i=1}^{M / 2}\left(\beta_{i}\left(\boldsymbol{\theta}_{1}\right)\right)^{2} \approx \sum_{i=1}^{M / 2}\left(\varphi_{i}\right)^{2} \\
& +\sum_{i=1}^{M / 2}\left(\beta_{i}\right)^{2}-2 \mu \sum_{i=1}^{M / 2} \beta_{i}\left(\mathbf{G}_{02}^{T} \frac{\partial \beta_{i}}{\partial \dot{\mathbf{u}}}\right) \\
& -2 \lambda \sum_{i=1}^{M / 2} \varphi_{i}\left(\mathbf{G}_{01}^{T} \frac{\partial \varphi_{i}}{\partial \mathbf{u}}\right)-2 \lambda \sum_{i=1}^{M / 2} \beta_{i}\left(\mathbf{G}_{01}^{T} \frac{\partial \beta_{i}}{\partial \mathbf{u}}\right) \\
& +\lambda^{2} \sum_{i=1}^{M / 2}\left(\mathbf{G}_{01}^{T} \frac{\partial \varphi_{i}}{\partial \mathbf{u}}\right)^{2}+\lambda^{2} \sum_{i=1}^{M / 2}\left(\mathbf{G}_{01}^{T} \frac{\partial \beta_{i}}{\partial \mathbf{u}}\right)^{2} \\
& +\left.\mu^{2} \sum_{i=1}^{M / 2}\left(\mathbf{G}_{02}^{T} \frac{\partial \beta_{i}}{\partial \dot{\mathbf{u}}}\right)^{2}\right|_{\boldsymbol{\theta}_{0}}
\end{aligned}
$$

Representing the gradient of $\rho\left(\boldsymbol{\theta}_{1}\right)$ with respect to $\lambda$ and $\mu$ satisfies the equation

$$
\begin{aligned}
& \frac{\partial \rho\left(\boldsymbol{\theta}_{1}\right)}{\partial \lambda}=0, \\
& \frac{\partial \rho\left(\boldsymbol{\theta}_{1}\right)}{\partial \mu}=0 .
\end{aligned}
$$

Thus, the optimal step size is

$$
\begin{aligned}
& \lambda \\
& =\left.\frac{\sum_{i=1}^{M / 2} \varphi_{i}\left(\mathbf{G}_{01}^{T}\left(\partial \varphi_{i} / \partial \mathbf{u}\right)\right)+\sum_{i=1}^{M / 2} \beta_{i}\left(\mathbf{G}_{01}^{T}\left(\partial \beta_{i} / \partial \mathbf{u}\right)\right)}{\sum_{i=1}^{M / 2}\left(\mathbf{G}_{01}^{T}\left(\partial \varphi_{i} / \partial \mathbf{u}\right)\right)^{2}+\sum_{i=1}^{M / 2}\left(\mathbf{G}_{01}^{T}\left(\partial \beta_{i} / \partial \mathbf{u}\right)\right)^{2}}\right|_{\boldsymbol{\theta}^{o}}, \\
& \mu=\left.\frac{\sum_{i=1}^{M / 2} \beta_{i}\left(\mathbf{G}_{02}^{T}\left(\partial \beta_{i} / \partial \dot{\mathbf{u}}\right)\right)}{\sum_{i=1}^{M / 2}\left(\mathbf{G}_{02}^{T}\left(\partial \beta_{i} / \partial \dot{\mathbf{u}}\right)\right)^{2}}\right|_{\boldsymbol{\theta}^{o}} .
\end{aligned}
$$

We have obtained the optimal step size which makes the convergence rate along the direction of negative gradient of objective function the fastest. Furthermore, a proper initial position and velocity are attained by (14) using (19) until (11) is sufficiently small.

2.3. Bias Compensation Using MLE. According to (8), the logarithm probability density function of the noise data $\mathbf{n}$ is

$$
\ln f(\mathbf{m}, \boldsymbol{\theta})=k-\frac{1}{2}[\mathbf{m}-\mathbf{F}(\boldsymbol{\theta})]^{T} \mathbf{Q}^{-1}[\mathbf{m}-\mathbf{F}(\boldsymbol{\theta})],
$$

where $k=-1 / 2 \ln \left((2 \pi)^{M}|\mathbf{Q}|\right)$ is constant and the MLE solution $\widehat{\boldsymbol{\theta}}$ is

$$
\widehat{\boldsymbol{\theta}}=\arg \max (I),
$$

where $I$ is the maximum likelihood cost function equal to

$$
I \triangleq-\frac{1}{2}[\mathbf{m}-\mathbf{F}(\boldsymbol{\theta})]^{T} \mathbf{Q}^{-1}[\mathbf{m}-\mathbf{F}(\boldsymbol{\theta})] .
$$

Due to the nonlinear form of $\mathbf{F}(\boldsymbol{\theta})$, iterative or exhaustive search method is required to find the ML solution $\widehat{\boldsymbol{\theta}}$ using the initial value of (14). Representing the gradient of $I$ with respect to $\boldsymbol{\theta}$ as $\mathbf{P}(\widehat{\boldsymbol{\theta}}), \widehat{\boldsymbol{\theta}}$ satisfies the equation

$$
\mathbf{P}(\widehat{\boldsymbol{\theta}})=\left.\frac{\partial I}{\partial \boldsymbol{\theta}}\right|_{\widehat{\boldsymbol{\theta}}}=0 .
$$

The expectation of the difference between $\widehat{\boldsymbol{\theta}}$ and $\boldsymbol{\theta}^{\circ}$ gives the bias $E\left[\widehat{\boldsymbol{\theta}}-\boldsymbol{\theta}^{o}\right]$. We use (23) to obtain the bias without explicitly solving $\widehat{\boldsymbol{\theta}}$. It assumes that the noise standard deviation relative to the true values of the TDOA and FDOA measurements is small. Thus, the noise terms higher than second order can be ignored, and the Taylor-series expansion of $\mathbf{P}(\widehat{\boldsymbol{\theta}})$ at $\boldsymbol{\theta}^{\circ}$ up to second order is

$$
\mathbf{P}(\widehat{\boldsymbol{\theta}})=\left.\frac{\partial I}{\partial \boldsymbol{\theta}}\right|_{\widehat{\boldsymbol{\theta}}} \approx \mathbf{H}^{\prime}+\mathbf{H}^{\prime \prime}\left(\widehat{\boldsymbol{\theta}}-\boldsymbol{\theta}^{o}\right)+\mathbf{g}\left(\boldsymbol{\theta}^{o}\right)=0,
$$

where

$$
\begin{aligned}
\mathbf{H}^{\prime} & =\left.\frac{\partial I}{\partial \boldsymbol{\theta}}\right|_{\boldsymbol{\theta}=\boldsymbol{\theta}^{o}}, \\
\mathbf{H}^{\prime \prime} & =\left.\frac{\partial^{2} I}{\partial \boldsymbol{\theta} \partial \boldsymbol{\theta}^{T}}\right|_{\boldsymbol{\theta}=\boldsymbol{\theta}^{o}}, \\
\mathbf{H}_{l}^{\prime \prime \prime} & =\left.\frac{\partial}{\partial \boldsymbol{\theta}_{l}}\left(\frac{\partial^{2} I}{\partial \boldsymbol{\theta} \partial \boldsymbol{\theta}^{T}}\right)\right|_{\boldsymbol{\theta}=\boldsymbol{\theta}^{o}} \quad l=1,2, \ldots, 6, \\
\mathbf{g}\left(\boldsymbol{\theta}^{o}\right) & =\frac{1}{2}\left[\begin{array}{c}
\operatorname{tr}\left(\mathbf{H}_{1}^{\prime \prime \prime} \times\left[\widehat{\boldsymbol{\theta}}-\boldsymbol{\theta}^{o}\right]\left[\widehat{\boldsymbol{\theta}}-\boldsymbol{\theta}^{o}\right]^{T}\right) \\
\operatorname{tr}\left(\mathbf{H}_{2}^{\prime \prime \prime} \times\left[\widehat{\boldsymbol{\theta}}-\boldsymbol{\theta}^{o}\right]\left[\widehat{\boldsymbol{\theta}}-\boldsymbol{\theta}^{o}\right]^{T}\right) \\
\operatorname{tr}\left(\mathbf{H}_{6}^{\prime \prime \prime} \times\left[\widehat{\boldsymbol{\theta}}-\boldsymbol{\theta}^{o}\right]\left[\widehat{\boldsymbol{\theta}}-\boldsymbol{\theta}^{o}\right]^{T}\right)
\end{array}\right]
\end{aligned}
$$

We can obtain the theoretical bias from (24):

$$
\begin{aligned}
E\left[\widehat{\boldsymbol{\theta}}-\boldsymbol{\theta}^{o}\right]= & E\left[-\left(\mathbf{H}^{\prime \prime}\right)^{-1} \mathbf{H}^{\prime}\right] \\
& +E\left[-\left(\mathbf{H}^{\prime \prime}\right)^{-1} \mathbf{g}\left(\boldsymbol{\theta}^{o}\right)\right] .
\end{aligned}
$$

Equation (25) is specifically expressed as

$$
\begin{aligned}
\mathbf{H}^{\prime} & =\left.\frac{\partial I}{\partial \boldsymbol{\theta}}\right|_{\boldsymbol{\theta}=\boldsymbol{\theta}^{o}}=\left.\frac{\partial^{T} \mathbf{F}(\boldsymbol{\theta})}{\partial \boldsymbol{\theta}} \mathbf{Q}^{-1} \mathbf{n}\right|_{\boldsymbol{\theta}=\boldsymbol{\theta}^{o}}=\mathbf{C}, \\
\mathbf{H}^{\prime \prime} & =\left.\frac{\partial^{2} I}{\partial \boldsymbol{\theta} \partial \boldsymbol{\theta}^{T}}\right|_{\boldsymbol{\theta}=\boldsymbol{\theta}^{o}}=-(\mathbf{A}-\mathbf{B}), \\
\mathbf{A} & =\left.\frac{\partial^{T} \mathbf{F}(\boldsymbol{\theta})}{\partial \boldsymbol{\theta}} \mathbf{Q}^{-1} \frac{\partial \mathbf{F}(\boldsymbol{\theta})}{\partial \boldsymbol{\theta}^{T}}\right|_{\boldsymbol{\theta}=\boldsymbol{\theta}^{o}}, \\
\mathbf{B} & =\left.\sum_{j=1}^{M} \sum_{i=1}^{M} q_{i j} n_{i} \frac{\partial^{2} F_{j}(\boldsymbol{\theta})}{\partial \boldsymbol{\theta} \partial \boldsymbol{\theta}^{T}}\right|_{\boldsymbol{\theta}=\boldsymbol{\theta}^{o}},
\end{aligned}
$$

where $q_{i j}$ is the element of $\mathbf{Q}^{-1}$. 
Next, we give a detailed derivation and obtain an algebraic solution of (26). According to (27), the first term of (26) can be approximated as

$$
\begin{aligned}
E\left[-\left(\mathbf{H}^{\prime \prime}\right)^{-1} \mathbf{H}^{\prime}\right] & =E\left[(\mathbf{A}-\mathbf{B})^{-1} \mathbf{C}\right] \\
& \approx E\left[\mathbf{A}^{-1} \mathbf{C}\right]+E\left[\mathbf{A}^{-1} \mathbf{B} \mathbf{A}^{-1} \mathbf{C}\right] .
\end{aligned}
$$

Because $\mathbf{A}$ does not contain noise, $E\left[\mathbf{A}^{-1} \mathbf{C}\right]=0$ and (27) can be simplified as

$$
E\left[-\left(\mathbf{H}^{\prime \prime}\right)^{-1} \mathbf{H}^{\prime}\right] \approx E\left[\mathbf{A}^{-1} \mathbf{B} \mathbf{A}^{-1} \mathbf{C}\right] .
$$

Substituting the definition of $\mathbf{A}, \mathbf{B}, \mathbf{C}$ from (27) and $E\left[n_{i} \mathbf{n}\right]=$ $\mathbf{Q e}_{i}$, the first component of bias is

$$
\begin{aligned}
E[ & \left.-\left(\mathbf{H}^{\prime \prime}\right)^{-1} \mathbf{H}^{\prime}\right] \\
& \approx \mathbf{A}^{-1} \sum_{i=1}^{M} \mathbf{P}_{i} \mathbf{A}^{-1}\left(\frac{\partial^{T} \mathbf{F}(\boldsymbol{\theta})}{\partial \boldsymbol{\theta}}\right) \mathbf{Q}^{-1} \cdot E\left[n_{i} \mathbf{n}\right] \\
& \left.\approx \mathbf{A}^{-1} \sum_{i=1}^{M} \mathbf{P}_{i} \mathbf{A}^{-1}\left(\frac{\partial^{T} \mathbf{F}(\boldsymbol{\theta})}{\partial \boldsymbol{\theta}}\right) \mathbf{e}_{i}\right|_{\boldsymbol{\theta}=\boldsymbol{\theta}^{o}},
\end{aligned}
$$

where $\mathbf{e}_{i}$ is $M \times 1$ zero vector except that its $i$ th element is unity. $\mathbf{P}_{i}$ is

$$
\mathbf{P}_{i}=\left.\sum_{j=1}^{M} q_{i j} \frac{\partial^{2} \mathbf{F}_{j}(\boldsymbol{\theta})}{\partial \boldsymbol{\theta} \partial \boldsymbol{\theta}^{T}}\right|_{\boldsymbol{\theta}=\boldsymbol{\theta}^{o}}
$$

The second bias component $E\left[-\left(\mathbf{H}^{\prime \prime}\right)^{-1} \mathbf{g}\left(\boldsymbol{\theta}^{o}\right)\right]$ is quite tedious to evaluate and we will approximate it [26]. When the noise level is small, from (27), we have $\mathbf{H}^{\prime \prime} \approx-\mathbf{A}$. Thus, the second bias component is

$$
E\left[-\left(\mathbf{H}^{\prime \prime}\right)^{-1} \mathbf{g}\left(\boldsymbol{\theta}^{o}\right)\right] \approx \mathbf{A}^{-1} \mathbf{z}
$$

where

$$
\mathbf{z} \triangleq E\left[\mathbf{g}\left(\boldsymbol{\theta}^{o}\right)\right] \approx \frac{1}{2}\left[\begin{array}{c}
\operatorname{tr}\left(E\left[\mathbf{H}_{1}^{\prime \prime \prime}\right] \times \operatorname{CRLB}\left(\boldsymbol{\theta}^{o}\right)\right) \\
\operatorname{tr}\left(E\left[\mathbf{H}_{2}^{\prime \prime \prime}\right] \times \operatorname{CRLB}\left(\boldsymbol{\theta}^{o}\right)\right) \\
\vdots \\
\operatorname{tr}\left(E\left[\mathbf{H}_{6}^{\prime \prime \prime}\right] \times \operatorname{CRLB}\left(\boldsymbol{\theta}^{o}\right)\right)
\end{array}\right] .
$$

$\operatorname{tr}(*)$ represents the trace operation and $\operatorname{CRLB}\left(\boldsymbol{\theta}^{\circ}\right)$ is the CRLB of $\boldsymbol{\theta}^{\circ}$ when its bias is neglected. The approximation is valid for small measurement noise and the fact that MLE is asymptotically efficient. In addition,

$$
E\left[\mathbf{H}_{l}^{\prime \prime \prime}\right]=\sum_{i=1}^{M}\left[\mathbf{h}_{i}^{T} \mathbf{e}_{l} \mathbf{P}_{i}+\mathbf{P}_{i} \mathbf{e}_{l} \mathbf{h}_{i}^{T}+\mathbf{h}_{i} \mathbf{e}_{l}^{T} \mathbf{P}_{i}^{T}\right],
$$

where

$$
\mathbf{h}_{i}=\left.\sum_{j=1}^{M} q_{i j} \frac{\partial \mathbf{F}_{j}(\boldsymbol{\theta})}{\partial \boldsymbol{\theta}}\right|_{\boldsymbol{\theta}=\boldsymbol{\theta}^{o}}
$$

Utilizing $\operatorname{CRLB}\left(\boldsymbol{\theta}^{\circ}\right)$ for a given positioning measurement type, $\mathbf{z}$ can be evaluated and the expectation of the bias is equal to

$$
\begin{aligned}
\mathbf{b} & =E\left[\widehat{\boldsymbol{\theta}}-\boldsymbol{\theta}^{o}\right] \\
& =\left.\mathbf{A}^{-1}\left(\sum_{i=1}^{M} \mathbf{P}_{i} \mathbf{A}^{-1}\left(\frac{\partial^{T} \mathbf{F}(\boldsymbol{\theta})}{\partial \boldsymbol{\theta}}\right) \mathbf{e}_{i}+\mathbf{z}\right)\right|_{\boldsymbol{\theta}=\boldsymbol{\theta}^{o}} .
\end{aligned}
$$

Equation (36) is the generic form of the bias and the complexity of computing bias is $O\left(M^{2}\right)$. When $\mathbf{F}(\boldsymbol{\theta})$ takes on different measurement types, the first and second derivatives will be different, yielding different amount of bias in the MLE solution. Appendices $\mathrm{A}$ and $\mathrm{B}$ give details about the evaluation of the derivatives for $\mathbf{F}(\boldsymbol{\theta})$.

Equation (36) can accurately predict the bias of the MLE; hence, the current source position and velocity after bias compensation are given by

$$
\widetilde{\boldsymbol{\theta}}=\widehat{\boldsymbol{\theta}}-\mathbf{b},
$$

where $\widetilde{\boldsymbol{\theta}}$ can approximately be treated as an unbiased estimator of $\boldsymbol{\theta}^{o}$ with covariance matrix $\operatorname{CRLB}\left(\boldsymbol{\theta}^{\circ}\right)$. The evolution of the bias in (36) requires the true value of target position and velocity which is not known in practice. We will replace the true value with the ML estimate.

In Section 4, we will present the computer simulation to corroborate our theoretical development and to compare the relative localization accuracy for different methods.

\section{The CRLB of Distributed Passive Sensors Localization under Gaussian Distribution}

The CRLB is the lowest possible variance that an unbiased linear estimator can achieve. The measurement vector $\boldsymbol{\theta}$ is Gaussian distributed. Hence, according to (20), the CRLB is equal to the inverse of the Fisher matrix [31] defined as

$$
\mathbf{J}=\left.E\left[\left(\frac{\partial \ln f(\mathbf{m}, \boldsymbol{\theta})}{\partial \boldsymbol{\theta}}\right)^{T}\left(\frac{\partial \ln f(\mathbf{m}, \boldsymbol{\theta})}{\partial \boldsymbol{\theta}}\right)\right]\right|_{\boldsymbol{\theta}=\boldsymbol{\theta}^{\circ}},
$$

where $\mathbf{m}=\left[r_{2,1}, \ldots, r_{M, M-1}, \dot{r}_{2,1}, \ldots, \dot{r}_{M, M-1}\right]^{T}$ is the vector of range and range rate difference from TDOA and FDOA measurements and $f(\mathbf{m}, \boldsymbol{\theta})$ is the probability density function of $\mathbf{m}$ that is parameterized by the vector $\boldsymbol{\theta}$. The partial derivative of $\ln f(\mathbf{m}, \boldsymbol{\theta})$ with respect to $\boldsymbol{\theta}$ is

$$
\frac{\partial \ln f(\mathbf{m}, \boldsymbol{\theta})}{\partial \boldsymbol{\theta}}=\left.\frac{\partial^{T} \mathbf{F}(\boldsymbol{\theta})}{\partial \boldsymbol{\theta}} \mathbf{Q}^{-1} \mathbf{n}\right|_{\boldsymbol{\theta}=\boldsymbol{\theta}^{o}}=\mathbf{C} .
$$

Thus, the CRLB for the underlying problem reduces to

$$
\begin{aligned}
\operatorname{CRLB}\left(\boldsymbol{\theta}^{o}\right) & =\mathbf{J}^{-1}=\left[\left.\left(\frac{\partial^{T} \mathbf{F}(\boldsymbol{\theta})}{\partial \boldsymbol{\theta}} \mathbf{Q}^{-1} \frac{\partial \mathbf{F}(\boldsymbol{\theta})}{\partial \boldsymbol{\theta}^{T}}\right)\right|_{\boldsymbol{\theta}=\boldsymbol{\theta}^{o}}\right]^{-1} \\
& =\left[\left.\mathbf{A}\right|_{\boldsymbol{\theta}=\boldsymbol{\theta}^{o}}\right]^{-1} .
\end{aligned}
$$


The details about the evaluation of the CRLB from (40) are shown in Appendix A.

The solution derivation is obtained by evaluating the bias and variance of the location estimate. Let $\widehat{\boldsymbol{\theta}}$ be the coarse location estimation that is obtained by MLE and let $\widetilde{\boldsymbol{\theta}}$ be a vector that contains the source position and velocity estimation after bias compensation. Thus, the two estimation results can be expressed as

$$
\begin{aligned}
& \widehat{\boldsymbol{\theta}}=\boldsymbol{\theta}^{o}+\Delta \widehat{\boldsymbol{\theta}}, \\
& \widetilde{\boldsymbol{\theta}}=\widehat{\boldsymbol{\theta}}-\mathbf{b},
\end{aligned}
$$

where $\Delta \widehat{\boldsymbol{\theta}}$ is the source location bias of $\widehat{\boldsymbol{\theta}}$. We can get the final source location bias:

$$
\Delta \widetilde{\boldsymbol{\theta}}=\Delta \widehat{\boldsymbol{\theta}}-\mathbf{b}
$$

The expectation of (42) gives the bias

$$
\begin{aligned}
E[\Delta \widetilde{\boldsymbol{\theta}}] & =E[\Delta \widehat{\boldsymbol{\theta}}-\mathbf{b}]=E\left[\left(\widehat{\boldsymbol{\theta}}-\boldsymbol{\theta}^{o}\right)-E\left(\widehat{\boldsymbol{\theta}}-\boldsymbol{\theta}^{o}\right)\right] \\
& =0
\end{aligned}
$$

where $\widetilde{\boldsymbol{\theta}}$ can approximately be treated as an unbiased estimator. Under the assumption explained before (24), the highorder noise terms in (24) tend to zero asymptotically as the measurement noise decreases so that $\mathbf{n}$ is zero mean asymptotically at the true value of $\widehat{\boldsymbol{\theta}}$. Therefore, from the MLE theory [31], $\widehat{\boldsymbol{\theta}}$ is asymptotically unbiased under the zero mean Gaussian noise but not unbiased estimator. Multiplying (42) by its transpose and taking expectation yields, the covariance of $\widetilde{\boldsymbol{\theta}}$ is

$$
\begin{aligned}
\operatorname{cov}(\widetilde{\boldsymbol{\theta}}) & =E\left[(\widetilde{\boldsymbol{\theta}}-E[\widetilde{\boldsymbol{\theta}}])(\widetilde{\boldsymbol{\theta}}-E[\widetilde{\boldsymbol{\theta}}])^{T}\right] \\
& =E\left[\Delta \widetilde{\boldsymbol{\theta}} \Delta \widetilde{\boldsymbol{\theta}}^{T}\right]=E\left[(\Delta \widehat{\boldsymbol{\theta}}-\mathbf{b})(\Delta \widehat{\boldsymbol{\theta}}-\mathbf{b})^{T}\right] \\
& =E\left[\Delta \widehat{\boldsymbol{\theta}} \Delta \widehat{\boldsymbol{\theta}}^{T}\right]-\mathbf{b} \mathbf{b}^{T},
\end{aligned}
$$

where $E\left[\Delta \widetilde{\boldsymbol{\theta}} \Delta \widetilde{\boldsymbol{\theta}}^{T}\right]$ and $E\left[\Delta \widehat{\boldsymbol{\theta}} \Delta \widehat{\boldsymbol{\theta}}^{T}\right]$ are the MSEM of $\widetilde{\boldsymbol{\theta}}$ and $\widehat{\boldsymbol{\theta}}$. Due to (43) and (44), the MSEM of $\widetilde{\boldsymbol{\theta}}$ is equal to the covariance of $\widetilde{\boldsymbol{\theta}}$ and, because of $\left(\mathbf{b b}^{T}\right)_{i i} \geq 0(i=1,2, \ldots, 6)$,

$$
\operatorname{cov}(\widetilde{\boldsymbol{\theta}})_{i i} \leq\left(E\left[\Delta \widehat{\boldsymbol{\theta}} \Delta \widehat{\boldsymbol{\theta}}^{T}\right]\right)_{i i}
$$

From (45), we can obtain that the covariance of the proposed method is smaller than that of MLE. Therefore, the bias compensation method is effective to reduce the bias of MLE and the performance of estimation after bias compensation is better than that of the original MLE.

\section{Simulation Results}

This section uses numerical simulations to demonstrate the proposed method and to compare its performance with other location estimators. The simulation scenario contains
TABLE 1: Nominal position (in meters) and velocities (in meters/ second) of receivers.

\begin{tabular}{lccccccc}
\hline Groups & $\begin{array}{c}\text { Receiver } \\
\text { number } i\end{array}$ & $x_{i}$ & $y_{i}$ & $z_{i}$ & $\dot{x}_{i}$ & $\dot{y}_{i}$ & $\dot{z}_{i}$ \\
\hline \multirow{2}{*}{ Set 1 } & 1 & -150 & -600 & 200 & 10 & 20 & -30 \\
& 2 & 50 & -750 & 200 & 20 & 30 & 0 \\
\multirow{2}{*}{ Set 2 } & 3 & 500 & -200 & 500 & -10 & 0 & 10 \\
& 4 & 600 & 100 & 600 & 10 & 20 & 15 \\
Set 3 & 5 & 100 & 600 & 800 & -10 & 20 & 20 \\
& 6 & -100 & 400 & 700 & 30 & 0 & 20 \\
Set 4 & 7 & -600 & 50 & 400 & 15 & 10 & -15 \\
& 8 & -750 & -100 & 500 & -20 & -15 & 10 \\
\hline
\end{tabular}

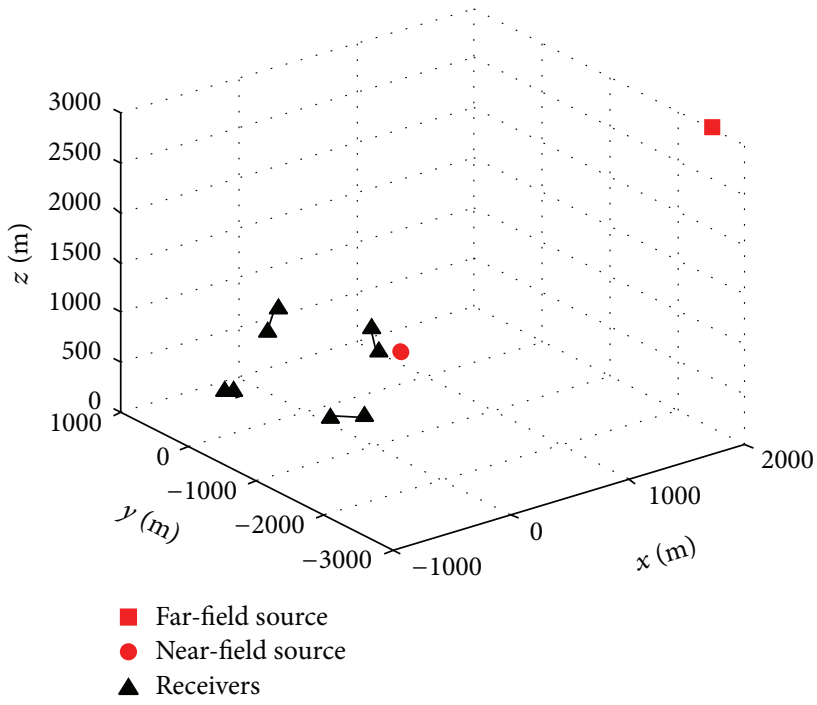

Figure 2: Localization geometry for simulation.

8 receivers (4 groups), and the position and velocity of receivers are given in Table 1 , as shown in Figure 2. TDOA and FDOA estimates were generated by adding to the true values zero mean Gaussian noise. The covariance matrices of TDOA and FDOA were $\sigma_{r}^{2} \mathbf{R}$ and $0.1 \sigma_{r}^{2} \mathbf{R}$, where $\mathbf{R}$ was set to 1 in the diagonal elements and 0.5 otherwise. $\sigma_{r}^{2}$ is the TDOA noise power multiplied by the square of the signal propagation speed $3 \times 10^{8} \mathrm{~m} / \mathrm{s}$ and the TDOA and FDOA noises were uncorrelated. The number of ensemble runs was $K=10000$. The estimation bias and accuracy are investigated for source as TDOA and FDOA estimates errors increase. The estimation accuracy in terms of the root mean square error (RMSE) is defined as

$$
\begin{aligned}
& \operatorname{RMSE}(\mathbf{u})=\sqrt{\frac{\sum_{k=1}^{K}\left\|\mathbf{u}_{k}-\mathbf{u}^{o}\right\|^{2}}{K}}, \\
& \operatorname{RMSE}(\dot{\mathbf{u}})=\sqrt{\frac{\sum_{k=1}^{K}\left\|\dot{\mathbf{u}}_{k}-\dot{\mathbf{u}}^{o}\right\|^{2}}{K}} .
\end{aligned}
$$



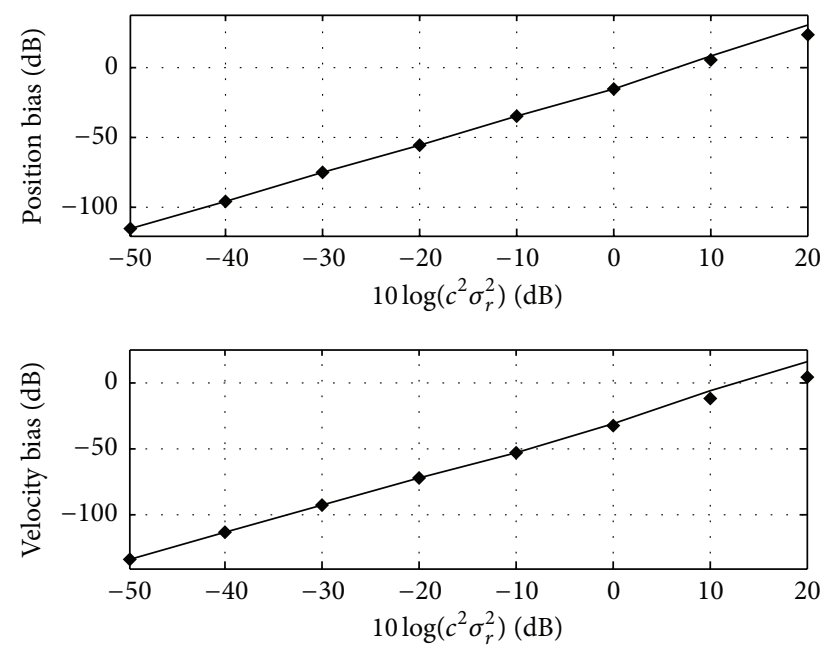

- Actual bias

- Theory bias

FIGURE 3: Comparison between theoretical and actual bias of estimation of source position and velocity by MLE for near-field source.

The estimation bias in terms of norm of estimation bias is defined as

$$
\begin{aligned}
& \operatorname{bias}(\mathbf{u})=\frac{1}{K}\left\|\sum_{k=1}^{K}\left(\mathbf{u}_{k}-\mathbf{u}^{o}\right)\right\|, \\
& \operatorname{bias}(\dot{\mathbf{u}})=\frac{1}{K}\left\|\sum_{k=1}^{K}\left(\dot{\mathbf{u}}_{k}-\dot{\mathbf{u}}^{o}\right)\right\|,
\end{aligned}
$$

where $\mathbf{u}^{o}$ and $\dot{\mathbf{u}}^{o}$ express the true position and velocity of the source and $\mathbf{u}_{k}$ and $\dot{\mathbf{u}}_{k}$ denote the estimated source position and velocity at ensemble $k$ and $K=10000$ is the number of ensemble runs. In particular, all the true values in the bias formula are replaced by the estimated and noisy measurement values.

4.1. For Near-Field Source. This section concerns near-field source localization. The true position and velocity of the source are $\mathbf{u}^{o}=[500,-500,600]^{T}$ and $\dot{\mathbf{u}}^{o}=[-30,-15,20]^{T}$. Figure 3 shows the comparison between theoretical bias and actual bias of estimation of target position and velocity by MLE. As shown in the figure, the theoretical bias (solid line) matches very well the simulation when the SNR is smaller than $10 \mathrm{~dB}$. With the increase of noise level, the theoretical bias value gradually deviates from the actual bias, especially the target velocity bias. This phenomenon in the proposed method is a consequence of ignoring the high-order terms of (24) in deriving the solution, which is not valid when the noise is large. Therefore, in order to obtain a more accurate estimation of target position, we should do the Taylor-series expansion of (24) at $\boldsymbol{\theta}^{\circ}$ up to high order.

Figure 4 shows the accuracy of position and velocity estimate of the proposed method in terms of RMSE as the noise level increases and compares it with the distributed
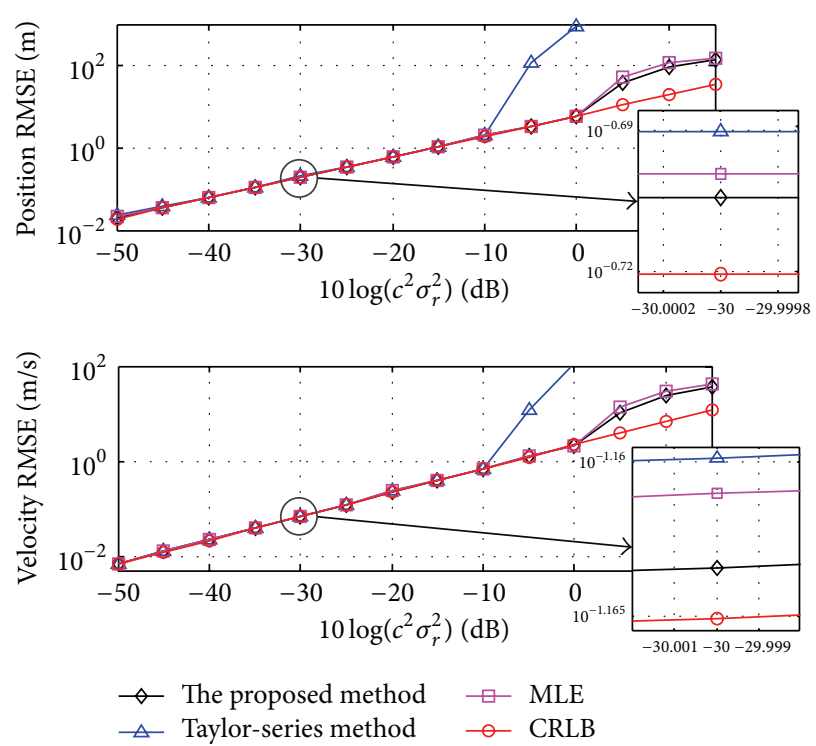

FIGURE 4: Comparison of root mean square error (RMSE) of the proposed method with the original MLE, Taylor-series method, and the CRLB for near-field source. The accuracy is shown in log scale as the noise power increase.

TDOA/FDOA localization algorithm MLE [26], Taylor-series method [10], and CRLB. The initial guess is obtained by SD method. The estimation precision of the proposed method is always higher than that of the other localization algorithms and all the algorithms can attain the CRLB at low to moderate noise level. The Taylor-series method deviates from the CRLB and gives an inaccurate solution at a noise power that is about $10 \mathrm{~dB}$ lower than the proposed technique. Furthermore, the new method achieves about $3.5 \mathrm{~dB}$ reduction in source position RMSE and $2.3 \mathrm{~dB}$ reduction in source velocity RMSE with respect to the one that does not account for the bias compensation when $\sigma_{r}^{2} \geq 1 \mathrm{~m}^{2}$. In the drawing of partial enlargement, the position and velocity estimation biases of the proposed estimators are all relatively small, which indicates that the proposed method exhibits the best performance. In addition, due to the nonlinear nature of this localization problem, the estimation accuracy of the three methods grows as the noise level increases.

In Figure 5, the estimation results clearly demonstrate that the bias of the proposed method is nonetheless smaller than the MLE for low noise level. The position and velocity biases of the proposed method are at least $40 \mathrm{~dB}$ and $35 \mathrm{~dB}$ lower than the MLE at low noise level. It is efficient to reduce the impact of the MLE bias on estimation. With the increase of the noise power, the original maximum likelihood estimation (MLE) is affected by the threshold effect, which leads to the decrease of the algorithm performance.

4.2. For Far-Field Source. This section concerns far-field source localization. The true position and velocity of the source are $\mathbf{u}^{o}=[2000,-2500,3000]^{T}$ and $\dot{\mathbf{u}}^{o}=[-30,-15$, $20]^{T}$. Figure 6 shows the comparison between theoretical and actual bias of estimation of target position and velocity by 

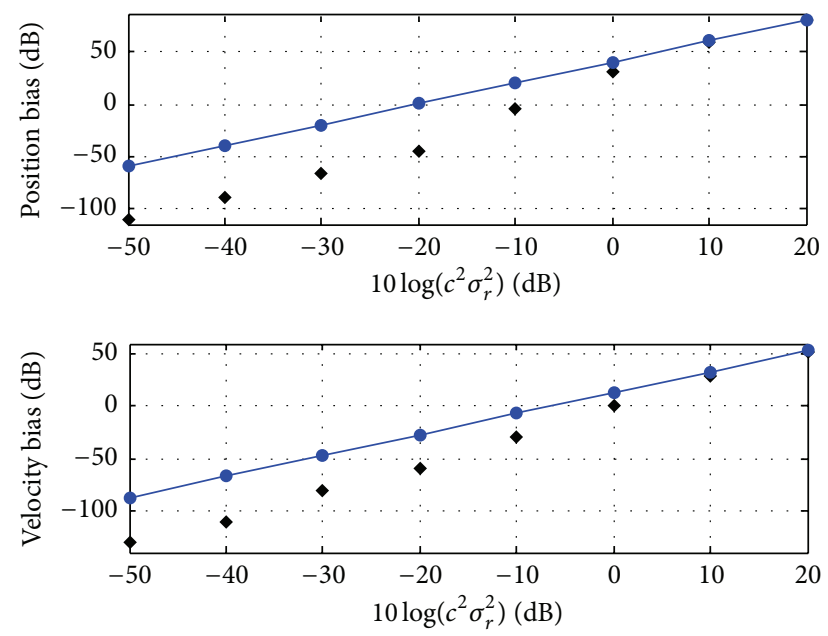

- The proposed method

$\rightarrow$ MLE

FIGURE 5: Comparison of the estimation bias of the proposed method with the original MLE against the Gaussian TDOA and FDOA noise for near-field source. The result is shown in log scale as the noise power increase.
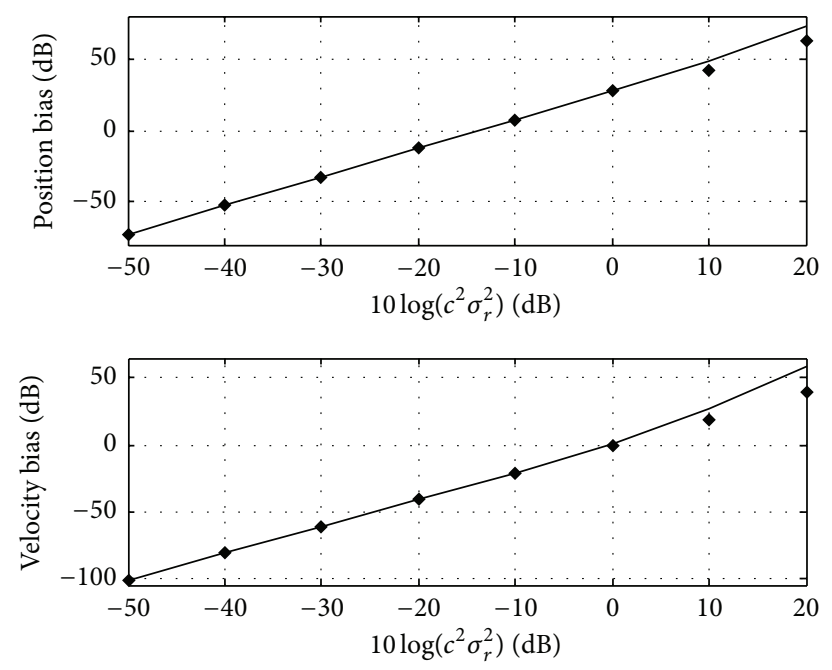

- Actual bias

- Theory bias

FIGURE 6: Comparison between theoretical and actual bias of estimation of source position and velocity by MLE for far-field source.

MLE. The trend of the result is the same as Figure 3 and the estimation results clearly indicate that theoretical bias of the far field is significantly close to the actual bias especially at low noise level.

Figure 7 shows the result for a far-field source about the accuracy of position and velocity estimate of the proposed method and other comparison algorithms in terms of RMSE
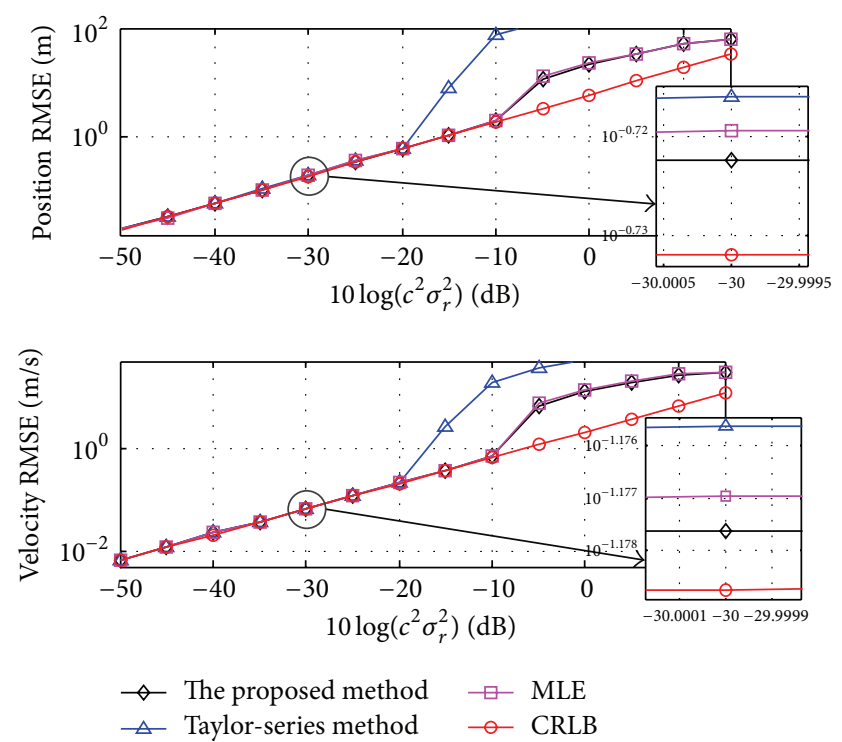

FIGURE 7: Comparison of root mean square error (RMSE) of the proposed method with the original MLE, Taylor-series method, and the CRLB for far-field source. The accuracy is shown in log scale as the noise power increase.

as the noise level increases. For far-field source, the following two conditions are satisfied:

$$
\begin{aligned}
& r_{1}^{o} \approx r_{2}^{o} \approx \cdots \approx r_{M}^{o}, \\
& \frac{\dot{r}_{1}^{o}}{r_{1}^{o}} \approx \cdots \approx \frac{\dot{r}_{M}^{o}}{r_{M}^{o}} \approx 0 .
\end{aligned}
$$

The first condition simply indicates that the ranges of the source to different receivers are approximately the same since the source is very far from the receivers. Due to the large range, the range rate relative to the range is close to zero. Thus, the thresholding effect of the far-field source occurs earlier than that of near-field source and the location accuracy is generally worse for a far-field source than a near-field source. As expected from the theory, the proposed solution meets the CRLB before the thresholding effect occurs at about $-10 \mathrm{~dB}$. Comparing with the MLE, the improvement of the proposed method is about $2.3 \mathrm{~dB}$ in the position and $1.3 \mathrm{~dB}$ in the velocity RMSEs and the estimation precision of the proposed method is always higher than that of the other comparison algorithms. Whatever the far-field and near-field sources, with the noise level decrease, the improvement of bias compensation is gradually reduced. This influence in the proposed method is caused by ignoring the high-order terms of (24) in deriving the solution.

Figure 8 shows the result for a far-field source about the bias analysis of the proposed method and the trend of the result is the same as Figure 5. Although the position and velocity bias of far-field source is about $5 \mathrm{~dB}$ smaller than that of the near-field source because of the two conditions shown in (48), the proposed method is still effective to reduce the bias of the MLE. 

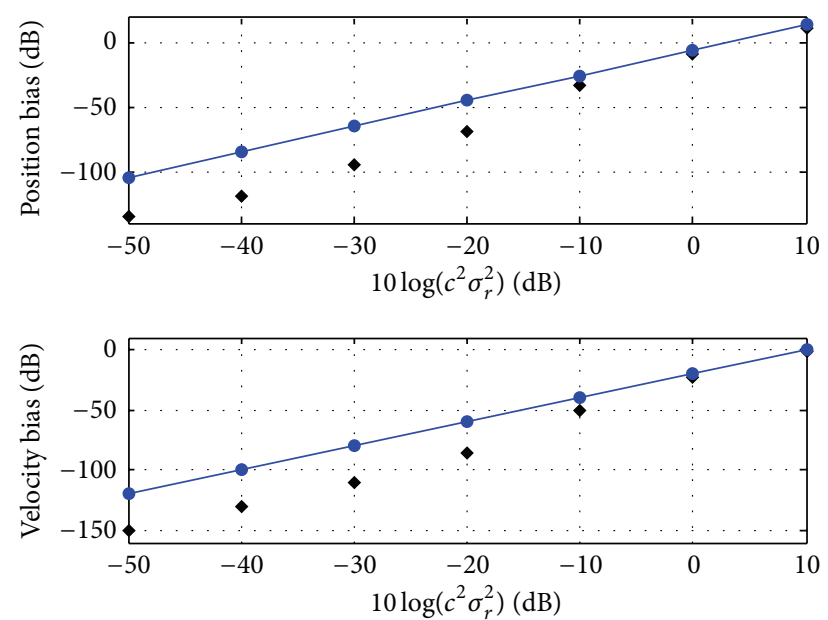

- The proposed method

$\rightarrow$ MLE

FIgURE 8: Comparison of the estimation bias of the proposed method with the original MLE against the Gaussian TDOA and FDOA noise for far-field source. The result is shown in log scale as the noise power increase.

\section{Conclusion}

Due to the disadvantages of centralized localization, we proposed a bias compensation algorithm based on MLE for distributed passive localization using TDOA and FDOA measurements which not only reduces the difficulty of the synchronization between the sensors, but also decreases the load of data computation and transmission. The proposed method tends to reduce the position and velocity bias of a moving source by using the steepest descent method and bias compensation of MLE. Computer simulation results show that the proposed method was able to achieve higher performance than the existing methods. The proposed solution does not require initial guess and attains the CRLB for Gaussian noise before the thresholding effect occurs. Meanwhile, small computation overhead and small memory demands make SD method and bias compensation method suitable for distributed localization schemes of wireless sensor networks. In addition, the position of each sensor should not be perfectly known (equip every sensor with a GPS receiver that is both energy and cost prohibitive). According to [37], we will take into account the position uncertainty of sensors for further study.

\section{Appendix}

\section{A. The First Derivatives of the $\mathrm{F}(\theta)$ for Source Position and Velocity}

The first derivatives of $\mathbf{F}(\boldsymbol{\theta})$ for source position and velocity are shown in this part. Let

$$
\begin{aligned}
\mathbf{r}(\boldsymbol{\theta}) & =\left[r_{2,1}(\boldsymbol{\theta}), r_{4,3}(\boldsymbol{\theta}), \ldots, r_{M, M-1}(\boldsymbol{\theta})\right]^{T}, \\
\dot{\mathbf{r}}(\boldsymbol{\theta}) & =\left[\dot{r}_{2,1}(\boldsymbol{\theta}), \dot{r}_{4,3}(\boldsymbol{\theta}), \ldots, \dot{r}_{M, M-1}(\boldsymbol{\theta})\right]^{T}, \\
\mathbf{F}(\boldsymbol{\theta}) & =\left[\mathbf{r}^{T}(\boldsymbol{\theta}), \dot{\mathbf{r}}^{T}(\boldsymbol{\theta})\right]^{T} .
\end{aligned}
$$

So $\partial \mathbf{F}(\boldsymbol{\theta}) / \partial \boldsymbol{\theta}^{T}$ in (27) can be expressed as

$$
\frac{\partial \mathbf{F}(\boldsymbol{\theta})}{\partial \boldsymbol{\theta}^{T}}=\left[\begin{array}{ll}
\frac{\partial \mathbf{r}(\boldsymbol{\theta})}{\partial \mathbf{u}^{T}} & \frac{\partial \mathbf{r}(\boldsymbol{\theta})}{\partial \dot{\mathbf{u}}^{T}} \\
\frac{\partial \dot{\mathbf{r}}(\boldsymbol{\theta})}{\partial \mathbf{u}^{T}} & \frac{\partial \dot{\mathbf{r}}(\boldsymbol{\theta})}{\partial \dot{\mathbf{u}}^{T}}
\end{array}\right] .
$$

According to (3) and (5), we let

$$
\begin{aligned}
& \mathbf{x}_{i}=r_{i}(\boldsymbol{\theta})^{-1}\left(\mathbf{u}-\mathbf{s}_{i}\right)^{T}, \\
& \mathbf{v}_{i}=r_{i}(\boldsymbol{\theta})^{-1}\left(\dot{\mathbf{u}}-\dot{\mathbf{s}}_{i}\right)^{T}-r_{i}(\boldsymbol{\theta})^{-2} \dot{r}_{i}(\boldsymbol{\theta})\left(\mathbf{u}-\mathbf{s}_{i}\right)^{T} .
\end{aligned}
$$

Thus, the partial derivatives of $\mathbf{r}(\boldsymbol{\theta})$ and $\mathbf{r}(\boldsymbol{\theta})$ with respect to $\mathbf{u}$ and $\dot{\mathbf{u}}$ yield (A.4), shown as

$$
\begin{gathered}
\frac{\partial \mathbf{r}(\boldsymbol{\theta})}{\partial \mathbf{u}^{T}}=\frac{\partial \dot{\mathbf{r}}(\boldsymbol{\theta})}{\partial \dot{\mathbf{u}}^{T}}=\left[\begin{array}{c}
\mathbf{x}_{2}-\mathbf{x}_{1} \\
\vdots \\
\mathbf{x}_{M}-\mathbf{x}_{M-1}
\end{array}\right]_{(M / 2) \times 3}, \\
\frac{\partial \dot{\mathbf{r}}(\boldsymbol{\theta})}{\partial \mathbf{u}^{T}}=\left[\begin{array}{c}
\mathbf{v}_{2}-\mathbf{v}_{1} \\
\vdots \\
\mathbf{v}_{M}-\mathbf{v}_{M-1}
\end{array}\right]_{(M / 2) \times 3}, \\
\frac{\partial \mathbf{r}(\boldsymbol{\theta})}{\partial \dot{\mathbf{u}}^{T}}=\mathbf{0}_{(M / 2) \times 3} .
\end{gathered}
$$

\section{B. The Second Derivatives of the $F(\theta)$ for Source Position and Velocity}

The second derivatives of $\mathbf{F}(\boldsymbol{\theta})$ for source position and velocity are shown in this part.

When $1 \leq j \leq M / 2, \partial^{2} \mathbf{F}_{j}(\boldsymbol{\theta}) / \partial \boldsymbol{\theta} \partial \boldsymbol{\theta}^{T}$ in (27) can be expressed as

$$
\frac{\partial^{2} \mathbf{F}_{j}(\boldsymbol{\theta})}{\partial \boldsymbol{\theta} \partial \boldsymbol{\theta}^{T}}=\left[\begin{array}{ll}
\frac{\partial^{2} \mathbf{r}_{2 j, 2 j-1}(\boldsymbol{\theta})}{\partial \mathbf{u} \partial \mathbf{u}^{T}} & \frac{\partial^{2} \mathbf{r}_{2 j, 2 j-1}(\boldsymbol{\theta})}{\partial \mathbf{u} \partial \dot{\mathbf{u}}^{T}} \\
\frac{\partial^{2} \mathbf{r}_{2 j, 2 j-1}(\boldsymbol{\theta})}{\partial \dot{\mathbf{u}} \partial \mathbf{u}^{T}} & \frac{\partial^{2} \mathbf{r}_{2 j, 2 j-1}(\boldsymbol{\theta})}{\partial \dot{\mathbf{u}} \partial \dot{\mathbf{u}}^{T}}
\end{array}\right]_{6 \times 6} .
$$

According to (3) and (5), we let

$$
\mathbf{X}_{j}=\left(\mathbf{I}-\mathbf{x}_{j}^{T} \mathbf{x}_{j}\right) r_{j}(\boldsymbol{\theta})^{-1},
$$

where I is the identity matrix. Therefore, the second-order partial derivatives of $\mathbf{r}(\boldsymbol{\theta})$ with respect to $\mathbf{u}$ and $\dot{\mathbf{u}}$ yield (B.3), shown as

$$
\begin{aligned}
\frac{\partial^{2} \mathbf{r}_{2 j, 2 j-1}(\boldsymbol{\theta})}{\partial \mathbf{u} \partial \mathbf{u}^{T}} & =\mathbf{X}_{2 j}-\mathbf{X}_{2 j-1}, \\
\frac{\partial^{2} \mathbf{r}_{2 j, 2 j-1}(\boldsymbol{\theta})}{\partial \dot{\mathbf{u}} \partial \mathbf{u}^{T}} & =\frac{\partial^{2} \mathbf{r}_{2 j, 2 j-1}(\boldsymbol{\theta})}{\partial \mathbf{u} \partial \dot{\mathbf{u}}^{T}}=\frac{\partial^{2} \mathbf{r}_{2 j, 2 j-1}(\boldsymbol{\theta})}{\partial \dot{\mathbf{u}} \partial \dot{\mathbf{u}}^{T}} \\
& =\mathbf{0}_{3 \times 3} .
\end{aligned}
$$


When $M / 2+1 \leq j \leq M, \partial^{2} \mathbf{F}_{j}(\boldsymbol{\theta}) / \partial \boldsymbol{\theta} \partial \boldsymbol{\theta}^{T}$ in (27) can be expressed as

$$
\begin{aligned}
& \frac{\partial^{2} \mathbf{F}_{j}(\boldsymbol{\theta})}{\partial \boldsymbol{\theta} \partial \boldsymbol{\theta}^{T}} \\
& \quad\left[\begin{array}{ll}
\frac{\partial^{2} \dot{\mathbf{r}}_{2 j-M, 2 j-1-M}(\boldsymbol{\theta})}{\partial \mathbf{u} \partial \mathbf{u}^{T}} & \frac{\partial^{2} \dot{\mathbf{r}}_{2 j-M, 2 j-1-M}(\boldsymbol{\theta})}{\partial \mathbf{u} \partial \dot{\mathbf{u}}^{T}} \\
\frac{\partial^{2} \dot{\mathbf{r}}_{2 j-M, 2 j-1-M}(\boldsymbol{\theta})}{\partial \dot{\mathbf{u}} \partial \mathbf{u}^{T}} & \frac{\partial^{2} \dot{\mathbf{r}}_{2 j-M, 2 j-1-M}(\boldsymbol{\theta})}{\partial \dot{\mathbf{u}} \partial \dot{\mathbf{u}}^{T}}
\end{array}\right]_{6 \times 6} .
\end{aligned}
$$

Let

$$
\begin{aligned}
& \mathbf{Y}_{j}=\dot{r}_{j}(\boldsymbol{\theta}) r_{j}(\boldsymbol{\theta})^{-2}\left(3 \mathbf{x}_{j}^{T} \mathbf{x}_{j}-\mathbf{I}\right), \\
& \mathbf{w}_{j}=r_{j}(\boldsymbol{\theta})^{-1}\left(\dot{\mathbf{u}}-\dot{\mathbf{s}}_{j}\right)^{T}, \\
& \mathbf{W}_{j}=r_{j}(\boldsymbol{\theta})^{-1} \mathbf{x}_{j}^{T} \mathbf{w}_{j}, \\
& \boldsymbol{\Psi}_{j}=\mathbf{Y}_{j}-\mathbf{W}_{j}-\mathbf{W}_{j}^{T} .
\end{aligned}
$$

The second-order partial derivatives of $\dot{\mathbf{r}}(\boldsymbol{\theta})$ with respect to $\mathbf{u}$ and $\dot{\mathbf{u}}$ yield (B.6), shown as

$$
\begin{aligned}
& \frac{\partial^{2} \dot{\mathbf{r}}_{2 j-M, 2 j-1-M}(\boldsymbol{\theta})}{\partial \mathbf{u} \partial \mathbf{u}^{T}}=\Psi_{2 j-M}-\Psi_{2 j-1-M}, \\
& \frac{\partial^{2} \dot{\mathbf{r}}_{2 j-M, 2 j-1-M}(\boldsymbol{\theta})}{\partial \dot{\mathbf{u}} \partial \mathbf{u}^{T}}=\mathbf{X}_{2 j-M}-\mathbf{X}_{2 j-1-M}, \\
& \frac{\partial^{2} \dot{\mathbf{r}}_{2 j-M, 2 j-1-M}(\boldsymbol{\theta})}{\partial \mathbf{u} \partial \dot{\mathbf{u}}^{T}}=\mathbf{X}_{2 j-M}-\mathbf{X}_{2 j-1-M}, \\
& \frac{\partial^{2} \dot{\mathbf{r}}_{2 j-M, 2 j-1-M}(\boldsymbol{\theta})}{\partial \dot{\mathbf{u}} \partial \dot{\mathbf{u}}^{T}}=\mathbf{0}_{3 \times 3} .
\end{aligned}
$$

\section{Competing Interests}

The authors declare that they have no competing interests.

\section{References}

[1] G. C. Carter, "Time delay estimation for passive sonar signal processing," IEEE Transactions on Acoustics, Speech, and Signal Processing, vol. 29, no. 3, pp. 463-470, 1981.

[2] E. Weinstein, "Optimal source localization and tracking from passive array measurements," IEEE Transactions on Acoustics, Speech, and Signal Processing, vol. 30, no. 1, pp. 69-76, 1982.

[3] T. Gustafsson, B. D. Rao, and M. Trivedi, "Source localization in reverberant environments: modeling and statistical analysis," IEEE Transactions on Speech and Audio Processing, vol. 11, no. 6, pp. 791-803, 2003.

[4] B. Mungamuru and P. Aarabi, "Enhanced sound localization," IEEE Transactions on Systems, Man, and Cybernetics, Part B: Cybernetics, vol. 34, no. 3, pp. 1526-1540, 2004.

[5] J. C. Chen, K. Yao, and R. E. Hudson, "Source localization and beamforming," IEEE Signal Processing Magazine, vol. 19, no. 2, pp. 30-39, 2002.
[6] T. S. Rappaport, J. H. Reed, and B. D. Woerner, "Position location using wireless communications on highways of the future," IEEE Communications Magazine, vol. 34, no. 10, pp. 33-41, 1996.

[7] A. Yeredor and E. Angel, "Joint TDOA and FDOA estimation: a conditional bound and its use for optimally weighted localization," IEEE Transactions on Signal Processing, vol. 59, no. 4, pp. 1612-1623, 2011.

[8] Y. T. Chan and K. C. Ho, "A simple and efficient estimator for hyperbolic location," IEEE Transactions on Signal Processing, vol. 42, no. 8, pp. 1905-1915, 1994.

[9] K. C. Ho and W. Xu, "An accurate algebraic solution for moving source location using TDOA and FDOA measurements," IEEE Transactions on Signal Processing, vol. 52, no. 9, pp. 2453-2463, 2004.

[10] W. H. Foy, "Position-location solutions by taylor-series estimation," IEEE Transactions on Aerospace and Electronic Systems, vol. AES-12, no. 2, pp. 187-194, 1976.

[11] R. O. Schmidt, "An algorithm for two-receiver TDOA/FDOA emitter location," Technical Memo TM-1229, ESL Incorporated, Sunnyvale, Calif, USA, 1980.

[12] K. C. Ho, X. Lu, and L. Kovavisaruch, "Source localization using TDOA and FDOA measurements in the presence of receiver location errors: analysis and solution," IEEE Transactions on Signal Processing, vol. 55, no. 2, pp. 684-696, 2007.

[13] X. Y. Sun, J. D. Li, P. Y. Huang, and J. Y. Pang, “Total least-squares solution of active target localization using TDOA and FDOA measurements in WSN," in Proceedings of the IEEE 22nd International Conference on Advanced Information Networking and Applications (AINAW '08), pp. 995-999, Okinawa, Japan, March 2008.

[14] K. Yang, J. P. An, X. Y. Bu, and G. C. Sun, "Constrained total least-squares location algorithm using time-difference-ofarrival measurements," IEEE Transactions on Vehicular Technology, vol. 59, no. 3, pp. 1558-1562, 2010.

[15] H. Yu, G. Huang, and J. Gao, "Constrained total least-squares localisation algorithm using time difference of arrival and frequency difference of arrival measurements with sensor location uncertainties," IET Radar, Sonar and Navigation, vol. 6, no. 9, pp. 891-899, 2012.

[16] W. Meng, W. Xiao, and L. Xie, "Optimal sensor pairing for TDOA based source localization in sensor networks," in Proceedings of the 8th International Conference on Information, Communications and Signal Processing (ICICS '11), pp. 1-5, IEEE, Singapore, December 2011.

[17] H. Seute, J. Grandin, C. Enderli, A. Khenchaf, and J. Cexus, "Why synchronization is a key issue in modern electronic support measures," in Proceedings of the 16th International Radar Symposium (IRS '15), pp. 794-799, IEEE, Dresden, Germany, June 2015.

[18] A. Yeredor, "On passive TDOA and FDOA localization using two sensors with no time or frequency synchronization," in Proceedings $f$ the IEEE International Conference on Acoustics, Speech and Signal Processing (ICASSP '13), pp. 4066-4070, IEEE, Vancouver, Canada, May 2013.

[19] J.-Y. Yoon, J.-W. Kim, W.-Y. Lee, and D.-S. Eom, "A TDoA-based localization using precise time-synchronization," in Proceedings of the 14th International Conference on Advanced Communication Technology (ICACT '12), pp. 1266-1271, PyeongChang, Republic of Korea, February 2012. 
[20] T. Li, A. Ekpenyong, and Y.-F. Huang, "Source localization and tracking using distributed asynchronous sensors," IEEE Transactions on Signal Processing, vol. 54, no. 10, pp. 3991-4003, 2006.

[21] W. Meng, L. Xie, and W. Xiao, "TDOA sensor pairing in multihop sensor networks," in Proceedings of the 11th ACM/IEEE Conference on Information Processing in Sensing Networks (IPSN '12), pp. 91-92, ACM, Beijing, China, April 2012.

[22] M. Pourhomayoun and M. L. Fowler, "Distributed computation for direct position determination emitter location," IEEE Transactions on Aerospace \& Electronic Systems, vol. 50, no. 4, pp. 2878-2889, 2014.

[23] W. Meng, L. Xie, and W. Xiao, "Decentralized TDOA sensor pairing in multihop wireless sensor networks," IEEE Signal Processing Letters, vol. 20, no. 2, pp. 181-184, 2013.

[24] H.-W. Wei, R. Peng, Q. Wan, Z.-X. Chen, and S.-F. Ye, "Multidimensional scaling analysis for passive moving target localization with TDOA and FDOA measurements," IEEE Trans actions on Signal Processing, vol. 58, no. 3, part 2, pp. 1677-1688, 2010.

[25] F. Meyer, E. Riegler, O. Hlinka, and F. Hlawatsch, "Simultaneous distributed sensor self-localization and target tracking using belief propagation and likelihood consensus," in Proceedings of the 46th Asilomar Conference on Signals, Systems and Computers (ASILOMAR '12), pp. 1212-1216, IEEE, Pacific Grove, Calif, USA, November 2012.

[26] L. Rui and K. C. Ho, "Bias analysis of source localization using the maximum likelihood estimator," in Proceedings of the IEEE International Conference on Acoustics, Speech, and Signal Processing (ICASSP '12), pp. 2605-2608, Kyoto, Japan, March 2012.

[27] L. Rui and K. C. Ho, "Bias compensation for target tracking from range based Maximum Likelihood position estimates," in Proceedings of the IEEE 7th Sensor Array and Multichannel Signal Processing Workshop (SAM '12), pp. 193-196, IEEE, Hoboken, NJ, USA, June 2012.

[28] R. J. Barton and D. Rao, "Performance capabilities of long-range UWB-IR TDOA localization systems," Eurasip Journal on Advances in Signal Processing, vol. 2008, Article ID 236791, 1 page, 2008.

[29] K. C. Ho, "Bias reduction for an explicit solution of source localization using TDOA," IEEE Transactions on Signal Processing, vol. 60, no. 5, pp. 2101-2114, 2012.

[30] B. Hao, Z. Li, P. Qi, and L. Guan, "Effective bias reduction methods for passive source localization using TDOA and GROA," Science China Information Sciences, vol. 56, no. 7, pp. 1-12, 2013.

[31] S. M. Kay, Fundamentals of Statistical Signal Processing, Estimation Theory, Prentice-Hall, Englewood Cliffs, NJ, USA, 1993.

[32] M. S. Bartlett, "Approximate confidence intervals. II. More than one unknown parameter," Biometrika, vol. 40, pp. 306-317, 1953.

[33] J. B. S. Haldane, "The estimation of two parameters from a sample," Sankhyā, vol. 12, no. 4, pp. 313-320, 1953.

[34] D. R. Cox and E. J. Snell, "A general definition of residuals," Journal of the Royal Statistical Society, Series B: Methodological, vol. 30, no. 2, pp. 248-275, 1968.

[35] T.-L. Zhang, "Solving non-linear equation based on steepest descent method," in Proceedings of the 4th International Conference on Information and Computing (ICIC '11), pp. 216-218, IEEE, April 2011.

[36] Q. Q. Shi, H. Huo, T. Fang, and R. D. Li, "Using steepest descent method to refine the node positions in wireless sensor networks," in Proceedings of the IET Conference on Wireless, Mobile and Sensor Networks (CCWMSN '07), pp. 1055-1058, Shanghai, China, December 2007.

[37] N. Wu, W. Yuan, H. Wang, and J. Kuang, “TOA-based passive localization of multiple targets with inaccurate receivers based on belief propagation on factor graph," Digital Signal Processing, vol. 49, pp. 14-23, 2016. 


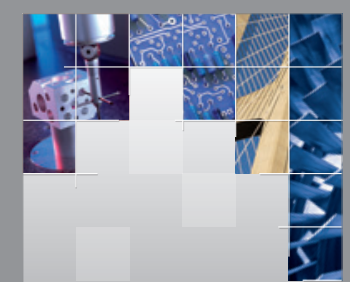

\section{Enfincering}
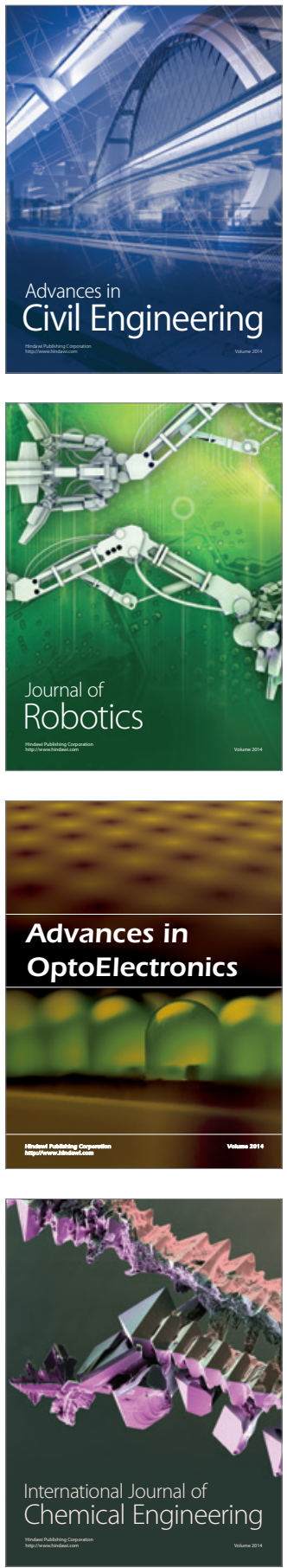

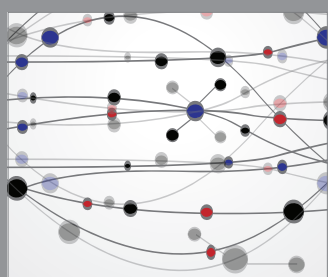

The Scientific World Journal

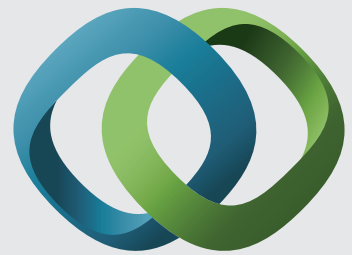

\section{Hindawi}

Submit your manuscripts at

http://www.hindawi.com
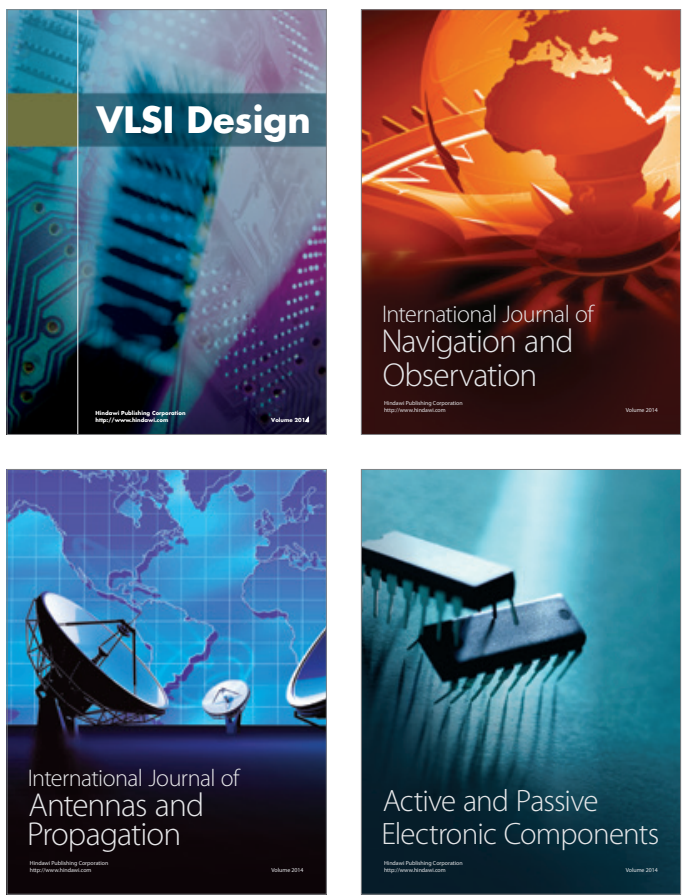
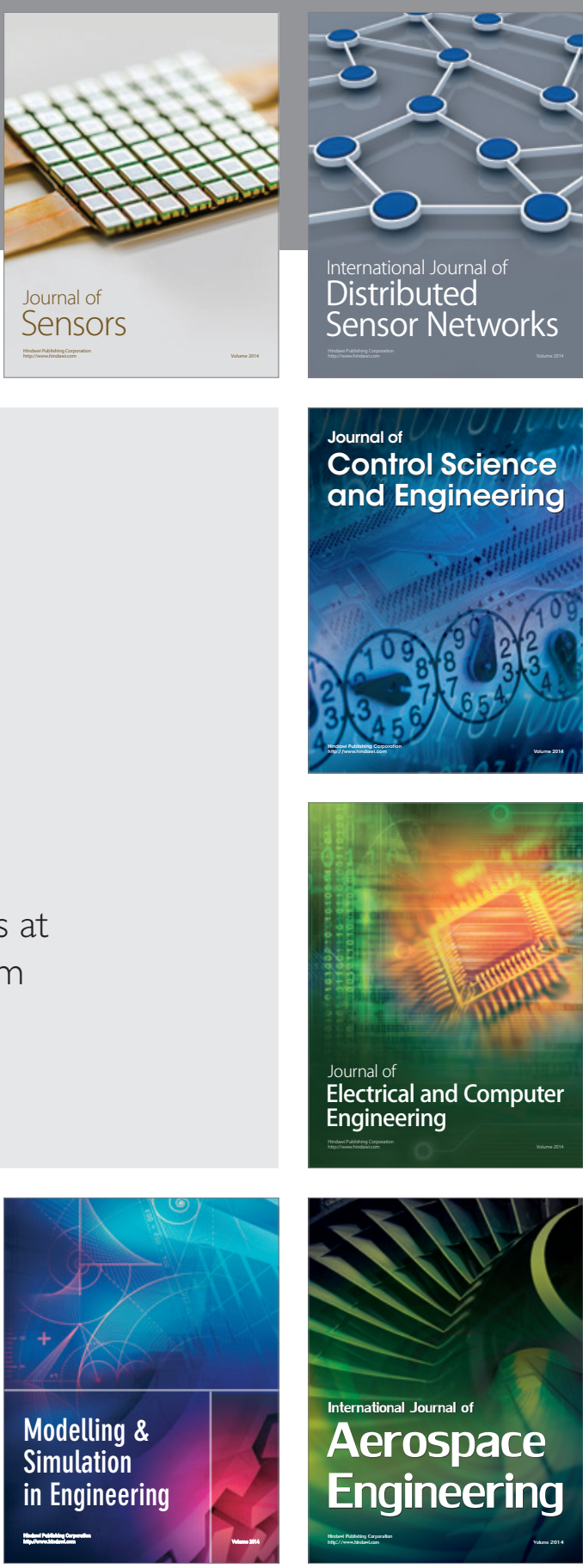

International Journal of

Distributed

Sensor Networks

Journal of

Control Science

and Engineering
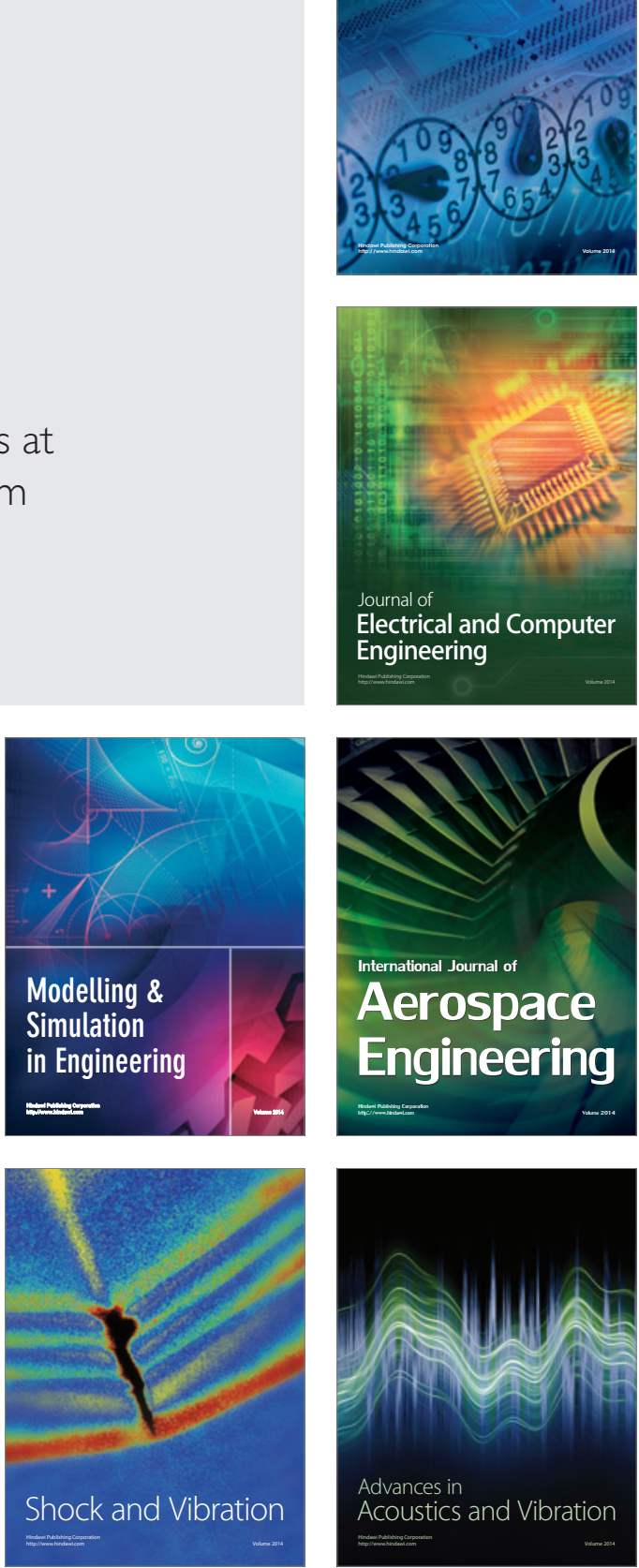